\title{
RINGS WHOSE ELEMENTS ARE SUMS OR MINUS SUMS OF THREE COMMUTING IDEMPOTENTS
}

\begin{abstract}
P. V. Danchev. Rings whose elements are sums or minus sums of three commuting idempotents, Mat. Stud. 49 (2018), 138-143.

We completely determine up to isomorphism those rings whose elements $x$ have the specific property that $x$ or $-x$ is a sum of three commuting idempotents. This statement strengthens well-known results in the subject due to Hirano-Tominaga (Bull. Austral. Math. Soc., 1988), Ying et al. (Can. Math. Bull., 2016), Tang et al. (Lin. \& Multilin. Algebra, 2018), Danchev (Boll. Un. Mat. Ital., 2019) and (Bull. Iran. Math. Soc., 2019).
\end{abstract}

1. Introduction and Background. Everywhere in the text of the present paper, all our rings $R$ are assumed to be associative, containing the identity element 1, which differs from the zero element 0 . Our standard terminology and notations are mainly in agreement with [10]. For instance, $J(R)$ stands for the Jacobson radical of $R, \operatorname{Nil}(R)$ stands for the set of all nilpotents in $R$ and $I d(R)$ stands for the set of all idempotents in $R$.

The leitmotif of the current article is to describe the isomorphic structure of all rings $R$ of the type that, for any $r \in R$, the element $r$ or $-r$ is a sum of three commuting idempotents; thereby generalizing the main result from [5], where only two commuting idempotents were considered. Obvious examples of such rings are the fields $\mathbb{Z}_{2}$ and $\mathbb{Z}_{3}$, the indecomposable ring $\mathbb{Z}_{4}$ and, in contrast to [12] and [11], the fields $\mathbb{Z}_{5}$ and $\mathbb{Z}_{7}$ occur as well.

Our motivation to do that is to enlarge in a very non-trivial way all of the following principally well-known results in the current subject by systematically developing a new approach. A brief retrospection of a most significant part of them is as follows: It is longer known that a Boolean ring is such a one whose elements are idempotents; this ring is a subdirect product of family of copies of the two element field $\mathbb{Z}_{2}$, i.e., it can be embedded in $\prod_{\lambda} \mathbb{Z}_{2}$ for some finite or infinite ordinal number $\lambda$. This was somewhat enlarged in [7] (see also [1]) to the so-called weakly Boolean rings in which each element is an idempotent or minus an idempotent. It was proved there that these rings are exactly either the Boolean ring $B$, or the three element field $\mathbb{Z}_{3}$, or the direct product $B \times \mathbb{Z}_{3}$ od two such rings.

On the other vein, in [9] were considered those rings whose elements are sums of two commuting idempotents; these rings are a subdirect product of families of copies of $\mathbb{Z}_{2}$ and $\mathbb{Z}_{3}$. This was recently somewhat extended in [5] to rings whose elements are sums or minus sums of two commuting idempotents. Besides, another generalization of the aforementioned result from [9] was given in [12] for rings in which each element is the sum or the difference of

2010 Mathematics Subject Classification: 13B99, 16E50, 16S34, 16U60, 16 U99.

Keywords: Boolean rings; finite fields; Jacobson radical; idempotents.

doi:10.15330/ms.49.2.138-143

(C) P. V. Danchev, 2018 
two commuting idempotents. However, the cited result from [5] somewhat encompasses that from [12].

On the other side, continuing the studies in this way, in [4] and [11] independently were described rings for which every element is a sum of three commuting idempotents. Moreover, in [4] was done even something more - rings whose elements are sums of three or differences of two commuting idempotents were completely described; thus improving also the already cited result from [12]. Likewise, in [3] a complete description of rings whose elements are sums of three or minus sums of two commuting idempotents were provided. Curiously, these two last sorts of rings do coincide.

Some other versions of rings which depend on at most four commuting idempotents, the interested readers can found in [2] and [6], respectively.

2. Main Results and Problems. We begin here with the following useful technicality.

Lemma 1. In any ring, in which each element is a sum or minus sum of three commuting idempotents, the relation $2^{2} .3 .5 .7=0$ holds.

Proof. Writing $4=e_{1}+e_{2}+e_{3}$ or $4=-e_{1}-e_{2}-e_{3}$ for some three commuting idempotents $e_{1}, e_{2}, e_{3}$, we deduce by multiplying both sides of these two equalities with $e_{1} e_{2} e_{3}$ that $e_{1} e_{2} e_{3}=0$ or that $7 e_{1} e_{2} e_{3}=0$, respectively. In the first case, the multiplication of the initial equality by $e_{1} e_{2}$ gives that $2 e_{1} e_{2}=0$ and, by a reason of symmetry, we obtain $2 e_{2} e_{3}=2 e_{3} e_{1}=0$. So, squaring now $4=e_{1}+e_{2}+e_{3}$ we get that $12=4.3=0$. Similar procedure for the second equality, which technical details we leave to the reader for a direct check, leads us to $42 e_{1} e_{2}=42 e_{2} e_{3}=42 e_{3} e_{1}=0$. Next, squaring $4=-e_{1}-e_{2}-e_{3}$, we derive that $20=2\left(e_{1} e_{2}+e_{2} e_{3}+e_{3} e_{1}\right)$ which, in turn, implies $20.21=4.3 .5 .7=0$, as claimed.

The next two assertions are rather helpful for our further applications, as the first of them is already proved and successfully used in [3]. They establish the curious facts that the only rings of characteristic 5 and 7 , respectively, in which all the elements are sums or minus sums of three commuting idempotents are the finite fields $\mathbb{Z}_{5}$ and $\mathbb{Z}_{7}$.

Proposition 1. ([3, Proposition 2.2 (i)]) Let $M$ be a ring of characteristic 5 whose elements satisfy the equations $x^{4}=1$ or $x^{3}=x$. Then $M \cong \mathbb{Z}_{5}$.

Proposition 2. Let $M$ be a ring of characteristic 7 whose elements satisfy the equations $x^{4}=x$ or $x^{4}=-x$. Then $M \cong \mathbb{Z}_{7}$.

Proof. Suppose $P$ is the subring of $M$, generated by its identity element. Hence, it is elementarily seen that $P \cong \mathbb{Z}_{7}$ as $7=0$. We intend to prove that $M=P$. To that goal, assume in a way of contradiction that there exists an element $b \in M \backslash P$. But we may also assume with no harm of generality that $b^{4}=-b$ since $b^{4}=b$ implies that $(3 b)^{4}=-(3 b)$ as $7=0$ and $3 b \notin P$ (for otherwise $3 b \in P$ yields that $6 b=-b \in P$ which is false). It is easily checked also that $(2 b)^{4}=-(2 b)$, because by assumption $b^{4}=-b$, where $2 b \notin P$ too (if not, $8 b=b \in P$, which is false). Let us now consider the case $(1+b)^{4}=1+b$. Hence $4 b^{3}+6 b^{2}+2 b=0$. We now claim that $(1+3 b)^{4} \neq \pm(1+3 b)$, however. Firstly, in the case of sign " + ", we get that $3 b^{3}+5 b^{2}-2 b=0$. Summing the two equations, we infer that $11 b^{2}=0$ as $7=0$. Thus $b^{2}=22 b^{2}=0$ and $b=0 \in P$ since $b^{4}=-b$, a contradiction. Secondly, in the case of sign "-", we get that $3 b^{3}+5 b^{2}+4 b+2=0$ which, in conjunction with $4 b^{3}+6 b^{2}+2 b=0$, ensures that $4 b^{2}-b+2=0$ taking into account once again that the characteristic of $M$ is 
exactly 7 . Thus, multiplying the lastly obtained equality by 2 , one infers that $b^{2}=2 b+3$ and substituting this in the equation $3 b^{3}+5 b^{2}+4 b+2=0$, one derives that $4 b^{3}=-4$, and hence $8 b^{3}=-8$, that is, $b^{3}=-1$, because as already used above $7=0$ in $M$. Now, we have two possibilities: $(1-b)^{4}=1-b$ or $(1-b)^{4}=-(1-b)$. The first equality leads to $6 b^{2}-4 b+4=0$ and since we already have $6 b^{2}+2 b-4=0$, these two equations imply $6 b=8$, that is, $b=-1=6 \in P$ which is manifestly false. The second equality leads to $3 b^{2}-3 b+3=0$ and since we already have $3 b^{2}+b-2=0$, these two equations yield $4 b=5$, i.e., $b=3 \in P$ which is obviously wrong.

That is why, it must be that $(1+b)^{4}=-(1+b)$ accomplished with $b^{4}=-b$. Consequently, $4 b^{3}+6 b^{2}+4 b+2=0$. Here, again $(1-b)^{4}=1-b$ or $(1-b)^{4}=-(1-b)=b-1$. Thus, in the first case, we arrive at $-4 b^{3}+6 b^{2}-4 b=0$ and the comparison of the existing two equations enables us that $b^{2}=1$. Hence $1=b^{4}=-b$ gives that $b=-1=6 \in P$ which is impossible. In the second case, we receive $4 b^{3}-6 b^{2}-b-2=0$ and the comparison of the two valid equations allows us to get that $b^{3}=-3 b$. So, $3 b^{2}=b$ and the substitution these two new relations in the initial equation $4 b^{3}+6 b^{2}+4 b+2=0$ assures that $6 b=2$ giving up that $b=-2=5 \in P$ which is impossible, too.

Finally, after all considerations, one concludes that such an element $b$ from $M$ does not exist outside $P$, and therefore the identity $M=P$ is really true, as promised.

We have now all the instruments necessary to prove the chief result which proof is hopefully based upon the assertions stated above.

Theorem 1. A non-zero ring $R$ possesses the property that all its elements are sums or minus sums of three commuting idempotents if, and only if, $R$ is a commutative ring such that $R \cong R_{1} \times R_{2} \times R_{3} \times R_{4}$, where $R_{1}, R_{2}, R_{3}, R_{4}$ are rings satisfying the next four conditions:

(1) $R_{1}=\{0\}$, or otherwise $R_{1}$ is a commutative ring in which $4=0, R_{1} / J\left(R_{1}\right)$ is a Boolean factor-ring and either $J\left(R_{1}\right)=\{0\}$ or $J\left(R_{1}\right)$ is a nilpotent ideal having the property that $J\left(R_{1}\right)=2 \operatorname{Id}\left(R_{1}\right)$.

(2) $R_{2}=\{0\}$, or $R_{2}$ is a subdirect product of isomorphic copies of the field $\mathbb{Z}_{3}$ otherwise.

(3) $R_{3}=\{0\}$ (which is mandatory when $R_{4}$ is non-zero), or $R_{3}$ is isomorphic to the field $\mathbb{Z}_{5}$ otherwise.

(4) $R_{4}=\{0\}$ (which is mandatory when $R_{3}$ is non-zero), or $R_{4}$ is isomorphic to the field $\mathbb{Z}_{7}$ otherwise.

Proof. Necessity. The classical Chinese Remainder Theorem applies to decompose $R \cong R_{1} \times$ $R_{2} \times R_{3} \times R_{4}$, where all of the rings $R_{1}, \cdots, R_{4}$ retain the same element-wise property as that of $R$ and in which the equalities $4=0,3=0,5=0$ and $7=0$ are fulfilled, respectively, too. We distinguish four basic cases thus:

Case 1: Describing $R_{1}$ : Since $4=2^{2}=0$, it follows that $2 \in J\left(R_{1}\right)$. Hence $R_{1} / J\left(R_{1}\right)$ is a quotient of characteristic 2 whose elements are sums of three idempotents, whence they are necessarily idempotents, as expected. That is why, $R_{1} / J\left(R_{1}\right)$ has to be Boolean.

What remains to show is the equality $J\left(R_{1}\right)=2 \operatorname{Id}\left(R_{1}\right)$. To do that, given $x \in J\left(R_{1}\right)$, it must be that $x=e_{1}+e_{2}+e_{3}$ or that $-x=e_{1}+e_{2}+e_{3}$ for some three commuting idempotents $e_{1}, e_{2}, e_{3} \in R_{1}$. Furthermore, after squaring the first equality, we come to $x^{2}=$ $x+2\left(e_{1} e_{2}+e_{2} e_{3}+e_{3} e_{1}\right)$ and thus to $2 x^{2}=2 x, x^{4}=x^{2}$ as $4=0$. In the second case $-x=e_{1}+e_{2}+e_{3}$, substituting $x \rightarrow-x$ we again arrive at the same equations for $x$ because $4=0$ and so $-2 x=2 x$. We therefore infer in both cases that $x^{2}\left(1-x^{2}\right)=0$ and that 
$2 x(1-x)=0$. But since both $1-x^{2}$ and $1-x$ invert in $R_{1}$, we conclude that $x^{2}=2 x=0$. Moreover, $x e_{1} e_{2} e_{3}=3 e_{1} e_{2} e_{3}=-e_{1} e_{2} e_{3}$ or $-x e_{1} e_{2} e_{3}=3 e_{1} e_{2} e_{3}=-e_{1} e_{2} e_{3}$. Consequently, $e_{1} e_{2} e_{3}(1 \pm x)=0$ and since $1 \pm x$ is invertible in $R_{1}$, it must be that $e_{1} e_{2} e_{3}=0$. But since $x^{2}=0$ and $4=0$, we then have $x=2\left(e_{1} e_{2}+e_{2} e_{3}+e_{3} e_{1}\right) \in 2 I d\left(R_{1}\right)$ because $e_{1} e_{2}+e_{2} e_{3}+e_{3} e_{1}$ is itself an idempotent, according to the fact that $e_{1} e_{2}, e_{2} e_{3}, e_{3} e_{1}$ are para-wise orthogonal idempotents which follows by taking into account the already established above equality that $e_{1} e_{2} e_{3}=0$. This substantiates our claim. In particular, $J\left(R_{1}\right)$ is even a nilpotent ideal and thus a nil-ideal with $J\left(R_{1}\right)^{2}=\{0\}$ as $4=0$.

Case 2: Describing $R_{2}$ : We intend to show that $R_{2} \subseteq \prod_{\mu} \mathbb{Z}_{3}$ for some finite or infinite ordinal $\mu$. Indeed, bearing in mind that $3=0$ in $R_{2}$ and any element $x$ here is the sum or minus the sum of three commuting idempotents, the equation $x^{3}=x$ is automatically valid utilizing to the classical binomial formula. Henceforth, we may apply [9] to get the wanted subdirect classification.

Case 3: Describing $R_{3}$ : We intend to prove that $R_{3} \cong \mathbb{Z}_{5}$. Here the equation $x^{5}=x$ is true for all elements $x \in R_{3}$, because $5=0$ in $R_{3}$. Writing $x=e+f+h$ for some three commuting idempotents $e, f, h$ in $R_{3}$, we detect via some long technical arguments, which direct check we leave to the readers, that the equation $3 x^{4}+2 x^{3}-2 x^{2}+2 x=0$ has to be satisfied by all elements of $R_{3}$ having the given record. In the case when $x=-e-f-h$, the substitution $x \rightarrow-x$ in the already obtained equation leads to $3 x^{4}-2 x^{3}-2 x^{2}-2 x=0$. Replacing now $x$ by $x-1$, we infer that $x^{4}=1$ in the first situation, or that $3 x^{4}+x^{3}+2 x^{2}-x=0$ in the second one. Let us now $y$ be an arbitrary element from $R_{3}$ such that $y^{4} \neq 1$. Hence it must satisfy the equation $3 y^{4}+y^{3}+2 y^{2}-y=0$. The replacement $x$ by $y^{3}$ in the final existing two equations for $x$, namely $x^{4}=1$ or $3 x^{4}+x^{3}+2 x^{2}-x=0$, enables us that $y^{4}=1$, which is against our choice, or that $3 y^{4}-y^{3}+2 y^{2}+y=0$ since $y^{5}=y$. The last equality in conjunction with $3 y^{4}+y^{3}+2 y^{2}-y=0$ ensures that $2 y^{3}=2 y$ which by multiplication with 3 guarantees that $y^{3}=y$ because $5=0$. We, therefore, can employ now Proposition 1 to conclude the wanted claim after all.

Case 4: Describing $R_{4}$ : We intend to prove that $R_{4} \cong \mathbb{Z}_{7}$. Here the equation $x^{7}=x$ is fulfilled for all elements $x \in R_{4}$, because $7=0$ in $R_{4}$. Writing $x=e+f+h$ for some three commuting idempotents $e, f, h$ in $R_{4}$, we extract via some long technical tricks, which we leave to the readers for a direct verification, that $x^{4}=x+e f h$. Thus $\left(x^{4}-x\right)^{2}=x^{4}-x$ and all elements of $R_{4}$ possessing the given record must satisfy the equation $2 x^{5}+x^{4}-2 x^{2}-x=0$. In the case when $x=-e-f-h$, the substitution $x \rightarrow-x$ in the already obtained equation leads to $2 x^{5}-x^{4}+2 x^{2}-x=0$. Just to note that, if an element $z$ satisfies simultaneously both equations, then it follows that $z^{4}=2 z^{2}$.

On the other hand, replacing $x$ by $2 x$ in the initial equations $2 x^{5}+x^{4}-2 x^{2}-x=0$ and $2 x^{5}-x^{4}+2 x^{2}-x=0$, we find that, for each element $x$, the equality $x^{5}+2 x^{4}-x^{2}-2 x=0$ or $x^{5}-2 x^{4}+x^{2}-2 x=0$ is valid. Hereafter, certain possible combinations must be fulfilled as follows: First Combination: $2 x^{5}+x^{4}-2 x^{2}-x=0$ along with $x^{5}+2 x^{4}-x^{2}-2 x=0$ give that $x^{4}=-x$. Second Combination: $2 x^{5}-x^{4}+2 x^{2}-x=0$ along with $x^{5}-2 x^{4}+x^{2}-2 x=0$ give that $x^{4}=-x$. Third Combination: $2 x^{5}+x^{4}-2 x^{2}-x=0$ along with $x^{5}-2 x^{4}+x^{2}-2 x=0$ give that $2 x^{4}+4 x^{2}-3 x=0$. Fourth Combination: $2 x^{5}-x^{4}+2 x^{2}-x=0$ along with $x^{5}+2 x^{4}-x^{2}-2 x=0$ give that $2 x^{4}+4 x^{2}+3 x=0$.

Let us now we replace $x$ by $3 x$ in the former equations $2 x^{5}+x^{4}-2 x^{2}-x=0$ and $2 x^{5}-x^{4}+2 x^{2}-x=0$. We thus find that for every element $x$ the equality $3 x^{5}+4 x^{4}-4 x^{2}-3 x=$ 0 or $3 x^{5}-4 x^{4}+4 x^{2}-3 x=0$ is true. 
We further differ a few more combinations like these:

- $2 x^{5}+x^{4}-2 x^{2}-x=0$ together with $3 x^{5}-4 x^{4}+4 x^{2}-3 x=0$ assure that $x^{4}=x$.

- $2 x^{5}-x^{4}+2 x^{2}-x=0$ together with $3 x^{5}-4 x^{4}+4 x^{2}-3 x=0$ assure that $x^{4}+x^{2}+2 x=0$. The last equality combined with $2 x^{4}+4 x^{2}+3 x=0$ imply that $x^{4}=x$, whereas being combined with $2 x^{4}+4 x^{2}-3 x=0$ they imply that $x^{4}=-2 x$. This, by squaring, yields that $3 x^{2}=0$ because $x^{7}=x$, whence $x^{2}=0$ and hence $x=0$.

- $2 x^{5}+x^{4}-2 x^{2}-x=0$ together with $3 x^{5}+4 x^{4}-4 x^{2}-3 x=0$ assure that $x^{4}+x^{2}-2 x=0$. The last equality combined with $2 x^{4}+4 x^{2}+3 x=0$ imply that $x^{4}=2 x$ which by squaring yields as above that $x=0$, while being combined with $2 x^{4}+4 x^{2}-3 x=0$ they imply that $x^{4}=-x$.

- $2 x^{5}-x^{4}+2 x^{2}-x=0$ together with $3 x^{5}+4 x^{4}-4 x^{2}-3 x=0$ assure that $x^{4}=-x$.

Finally, all of these possibilities allow us to conclude that $x^{4}=x$ or $x^{4}=-x$. Consequently, Proposition 2 is applicable to deduce that the desired claim is true after all.

In closing, what suffices to show is that the direct product $\mathbb{Z}_{5} \times \mathbb{Z}_{7}$ does not lie in the class determined by $R$. To do that, we consider the element $(1,4)$, where $1 \in \mathbb{Z}_{5}$ and $4 \in \mathbb{Z}_{7}$. Sufficiency. Treating the commutative ring $R_{1}$, it easily follows that for every $r_{1} \in R_{1}$ we have $r_{1}-r_{1}^{2} \in J\left(R_{1}\right)$ and since $J\left(R_{1}\right)$ is nil, there exists an idempotent $g \in R_{1}$ such that $r_{1}-g \in J\left(R_{1}\right)=2 I d\left(R_{1}\right)$. Therefore, although it is somewhat quite surprising, it is immediate that $r_{1}$ is a sum of three idempotents, as expected.

Dealing with the commutative ring $R_{2}$, it was shown in [9] that each element from it is a sum of three idempotents.

Concentrating on the finite commutative ring $R_{3}=\{0,1,2,3,4 \mid 5=0\}$, one sees that the element 1 is only a sum of three idempotents, whereas the elements 0,2 and 3 are simultaneously sums and minus sums of three idempotents, and 4 is minus an idempotent.

As for the finite commutative ring $R_{4}=\{0,1,2,3,4,5,6 \mid 7=0\}$, one observes that the elements 1, 2 and 3 are only sums of three idempotents, while the elements 4,5 and 6 are minus sums of three idempotents. The only existing element which is simultaneously a sum or a minus sum of idempotents is the 0 .

In particular, it is not too hard to verify that direct products of the kind $R_{1} \times R_{2} \times R_{3}$ and $R_{1} \times R_{2} \times R_{4}$, where certainly any of $R_{1}, R_{2}, R_{3}$ could be zero as well, possess the required idempotent property, because of the specific properties of each of the components in the direct products.

The considerations alluded to above lead us to the following discussion:

Remark 1. As the proof of the theorem unambiguously demonstrates, the direct products $\mathbb{Z}_{5} \times \mathbb{Z}_{5}$ and $\mathbb{Z}_{7} \times \mathbb{Z}_{7}$ do not have the required property, although separately each of the direct factors $\mathbb{Z}_{5}$ and $\mathbb{Z}_{7}$ has it. Same holds and for the direct product $\mathbb{Z}_{5} \times \mathbb{Z}_{7}$, so that the requirements about $R_{3}=\{0\}$ when $R_{4} \neq\{0\}$ and $R_{4}=\{0\}$ when $R_{3} \neq\{0\}$ are essential and cannot be dropped off. In fact, as already indicated above, the element $(1,4)$ in $\mathbb{Z}_{5} \times \mathbb{Z}_{7}$ is not presentable in the needed form, so that it substantiates our claim.

However, a routine check illustrates that the direct products $\mathbb{Z}_{3} \times \mathbb{Z}_{4}, \mathbb{Z}_{3} \times \mathbb{Z}_{5}$ and $\mathbb{Z}_{3} \times \mathbb{Z}_{7}$ as well as $\mathbb{Z}_{4} \times \mathbb{Z}_{5}$ and $\mathbb{Z}_{4} \times \mathbb{Z}_{7}$ lie in the considered class of rings.

In this direction, the next example sheds some more light on the chief result quoted above. 
Example 1. Although it is somewhat rather strange, the triple direct products $\mathbb{Z}_{4} \times \mathbb{Z}_{3} \times \mathbb{Z}_{5}$ and $\mathbb{Z}_{4} \times \mathbb{Z}_{3} \times \mathbb{Z}_{7}$ possess the properties stated in Theorem 1 . Even something more, because of the fact that $R_{1}$ and $R_{2}$ could be infinite instead of $R_{3}$ and $R_{4}$, we may also form infinite direct products of the above types, where the number of the copies isomorphic to $\mathbb{Z}_{4}$ and $\mathbb{Z}_{3}$ would be an arbitrary infinite ordinal. Concerning the two element field $\mathbb{Z}_{2}$, it also can be included in such direct products since the signs "+" and "-_" are tantamount.

In conclusion, let us resume that we were involved above with rings $R$ in which all elements $r$ were written as $r=e_{1}+e_{2}+e_{3}$ or $r=-e_{1}-e_{2}-e_{3}$. In this aspect, we end our work with the following two partial problems of some interest and importance:

Problem 2. Classify the isomorphic structure of those rings $R$ whose all elements $r$ are represented as $r=e_{1}+e_{2}+e_{3}$ or $r=e_{1}-e_{2}-e_{3}$.

Problem 3. Classify the isomorphic structure of those rings $R$ whose all elements $r$ are represented as $r=e_{1}+e_{2}+e_{3}$ or $r=e_{1}+e_{2}-e_{3}$.

In addition, our final important query, that immediately arises from the considerations alluded to above, is of whether or not a ring is commutative, provided that its elements are sums or linear combinations (thus including the sign "_-" as well) of a finite number of commuting idempotents (in particular, even if this number is fixed)? We conjecture that the answer is definitely "yes".

For a more account to this question, the interested reader can see also cf. [8].

\section{REFERENCES}

1. M.-S. Ahn, D.D. Anderson, Weakly clean rings and almost clean rings, Rocky Mountain J. Math., 36 (2006), 783-798.

2. P.V. Danchev, Rings whose elements are represented by at most three commuting idempotents, Gulf J. Math., 6 (2018), 1-6.

3. P.V. Danchev, Rings whose elements are sums of three or minus sums of two commuting idempotents, Alban. J. Math., 12 (2018), 3-7.

4. P.V. Danchev, Rings whose elements are sums of three or differences of two commuting idempotents, Bull. Iran. Math. Soc., 45 (2019).

5. P.V. Danchev, Rings whose elements are sums or minus sums of two commuting idempotents, Boll. Un. Mat. Ital., 12 (2019).

6. P.V. Danchev, Rings whose elements are sums of four commuting idempotents, to appear.

7. P.V. Danchev, W.Wm. McGovern, Commutative weakly nil clean unital rings, J. Algebra, 425 (2015), 410-422.

8. P.V. Danchev, E. Nasibi, The idempotent sum number and n-thin unital rings, Ann. Univ. Sci. Budapest, (Sect. Math.), 59 (2016), 85-98.

9. Y. Hirano, H. Tominaga, Rings in which every element is the sum of two idempotents, Bull. Austral. Math. Soc., 37 (1988), 161-164.

10. T.Y. Lam, A First Course in Noncommutative Rings, Second Edition, Graduate Texts in Math., Vol. 131, Springer-Verlag, Berlin-Heidelberg-New York, 2001.

11. G. Tang, Y. Zhou, H. Su, Matrices over a commutative ring as sums of three idempotents or three involutions, Lin. and Multilin. Algebra (2018).

12. Z. Ying, T. Koşan, Y. Zhou, Rings in which every element is a sum of two tripotents, Can. Math. Bull., 59 (2016), №3, 661-672.

Institute of Mathematics and Informatics, Bulgarian Academy of Sciences

Sofia, Bulgaria

danchev@math.bas.bg,pvdanchev@yahoo.com 